

\title{
Rinnovare il patrimonio. Learning from contemporary artists
}

Renew the legacy. Learning from contemporary artists

\begin{abstract}
Three contemporary art projects suggest some useful reflections to introduce a discontinuity in the ways of thinking about the transformation of existing spaces going beyond the misunderstood oppositions as "old" and "new". In Trentino, the artworks by Collective OP, Anna Scalfi Eghenter and Michele De Lucchi show how art and culture are extraordinary means for social innovation but also for promoting new architectural practices based on reduce, reuse and recycle that may be kept in mind for the development of further initiatives.
\end{abstract}

\section{Gianluca Cepollaro}

He's director of the School for Spatial and Landscape Management (TSM - STEP) in Trento, Italy. His research focuses on the areas of landscape and environmental education, the management of educational institutions and development processes of work and organisations.
Keywords

Landscape, contemporary art, architectural practice, reduce-reuserecycle, heritage. 
L'ormai condivisa centralità del riuso, del recupero, della rigenerazione e del riciclo per la progettazione architettonica può trarre, per l'innovazione degli approcci e dei metodi, numerosi spunti dalla ricerca dell'arte contemporanea.

In Trentino alcuni progetti degli ultimi due anni sembrano suggerire interessanti riflessioni particolarmente utili per introdurre una discontinuità nei modi di pensare e praticare la trasformazione degli spazi esistenti andando oltre le malintese dicotomie, prima tra tutte quella del "vecchio" e "nuovo", ma anche come vedremo del "pieno" e "vuoto", del "visibile" e "invisibile", del "sotto" e "sopra", del "dentro" e "fuori". Si tratta di iniziative eterogenee accomunate dal fatto di essere tese al superamento di un modo tradizionale di guardare al territorio. Sono progetti prima di tutto capaci di confrontarsi con alcuni temi rilevanti per vivere il tempo attuale quali la capacità di sintesi tra memoria e presente, la tutela dell'ambiente, la gestione delle risorse naturali, la valorizzazione del paesaggio, la cura per la vivibilità dei luoghi, lo sviluppo di forme di convivenza inclusive contro il crescente degrado di civiltà.

Il principale problema del modo di confrontarci con la necessaria innovazione della progettazione architettonica risiede probabilmente nell'incapacità di abbandonare logiche del tipo aut ... aut per imboccare le vie dell'et ... et, ma anche nell'inaridimento dell'immaginario e nella difficoltà di creare visioni in grado di anticipare il futuro. Per questo l'arte e la creatività sembrano essere la strada maestra per produrre quel salto, quello scatto in avanti o forse di lato, che è innanzitutto culturale. È solo attraverso l'arte, aprendoci alle sollecitazioni del gesto creativo, che riusciamo a rintracciare nuove connessioni, altre relazioni, e quindi ci rendiamo disponibili a cercare modi di intendere, pensare e vivere alternativi rispetto a quelli che fino a un momento prima ci apparivano come egemoni.

Un esempio straordinario, che non sarà però approfondito in queste pagine ma che si muove in questa direzione, è il lavoro che il giovane artista lombardo Edoardo Tresoldi ha realizzato in $\mathrm{Pu}-$ glia nel parco archeologico di Siponto. Utilizzan- do la rete metallica come materia prima sono stati ricostruiti, in scala reale, i volumi di una basilica paleocristiana partendo dai resti di parte della pavimentazione a mosaico e del perimetro murario originario. L'imponente e suggestiva installazione, che si eleva fino ai 14 metri di altezza, è di fatto un'architettura leggera e trasparente, costruita ar-

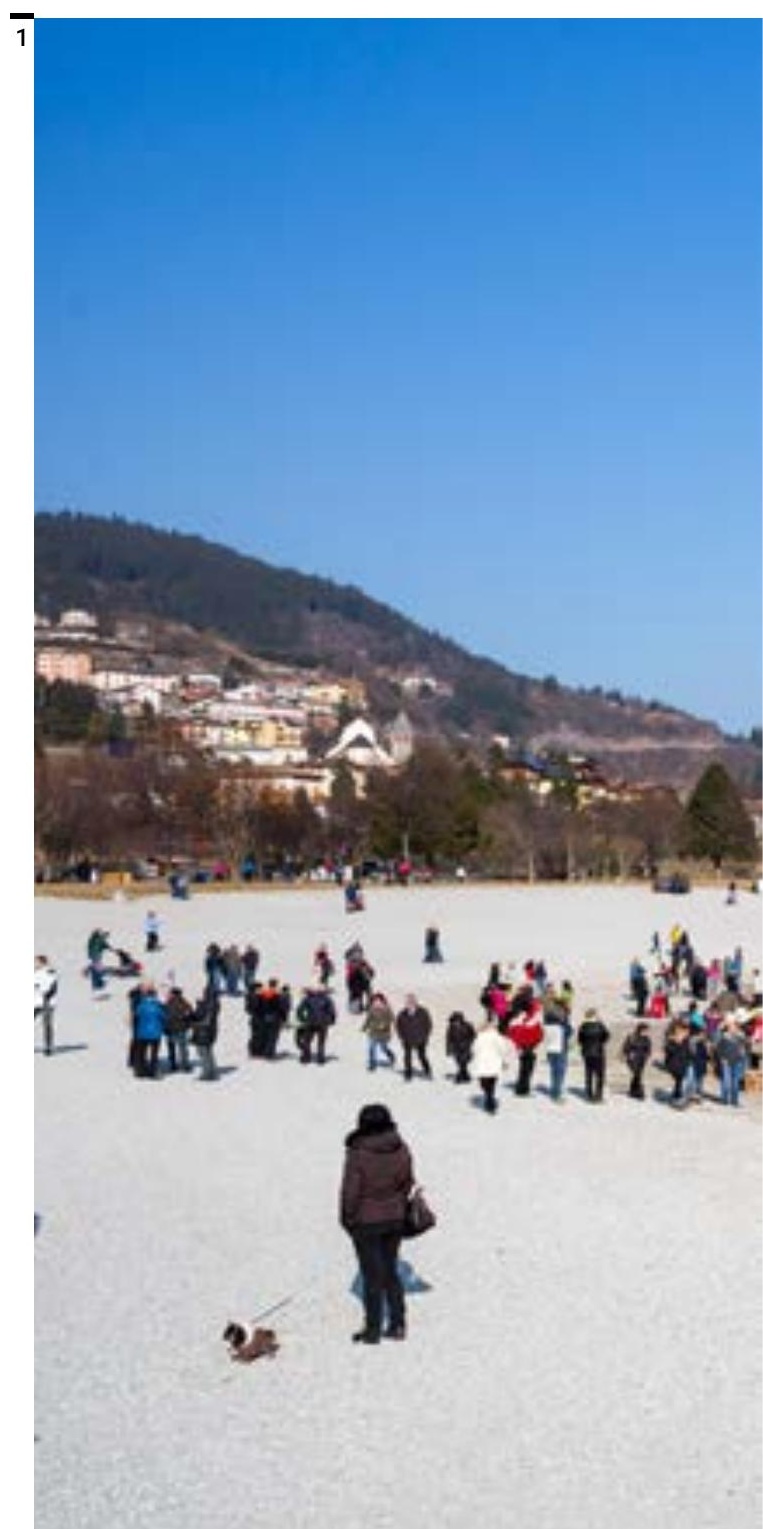


tigianalmente, che può segnare una svolta per la conservazione e la valorizzazione del patrimonio archeologico aprendo a nuovi ed inediti scenari. Analogamente all'opera di Tresoldi, tre lavori realizzati in Trentino ci segnalano la strada per la ricerca di una maggiore consapevolezza delle opportunità emergenti dai processi di riciclo, recupero, rigenerazione e riuso del costruito.

\section{Pieno/vuoto. "IO. Sempre". Il Collettivo OP al lago di Molveno}

Durante l'inverno tra il 2016 e il 2017 il lago di

Fig. 1 Molveno ha subito uno svuotamento delle proprie Il tavolo acque per alcuni lavori di manutenzione degli imdell'interazione sulle sponde del lago di Molveno svuotato (foto Filippo Frizzera). pianti per la produzione di energia idroelettrica. Il lago svuotato, in attesa di essere riempito nuovamente, appare subito lontano dalle narrazioni consuete, ad un uso in primo luogo ricreativo. $\mathrm{Al}$ contempo si mostra in modo assai più prossimo alla sua lunga storia. Eppure è sempre lui! Un lago che non è solo "natura" incontaminata, così come nell'immaginario sedimentato del turismo, ma che è stato oggetto di interventi trasformativi e che si presenta come esito temporaneo di un processo di lunga durata inestricabilmente naturale e culturale. Lo svuotamento del lago minaccia con forza l'immaginario sedimentato e tradizionale su cui si fonda l'economia turistica, genera ansia e paura. Nel momento in cui svaniscono tutte quelle sicurezze costruite dalla modernità, considerate ai nostri occhi date, fisse ed immutabili, possiamo però scoprire la possibilità di mettere in discussione ciò che ci appare scontato aprendoci a nuovi significati e possibilità inesplorate (De Rossi, 2018). Il lago vuoto, con il suo fondo calpestabile, per essere compreso e vissuto in modo non usuale richiede

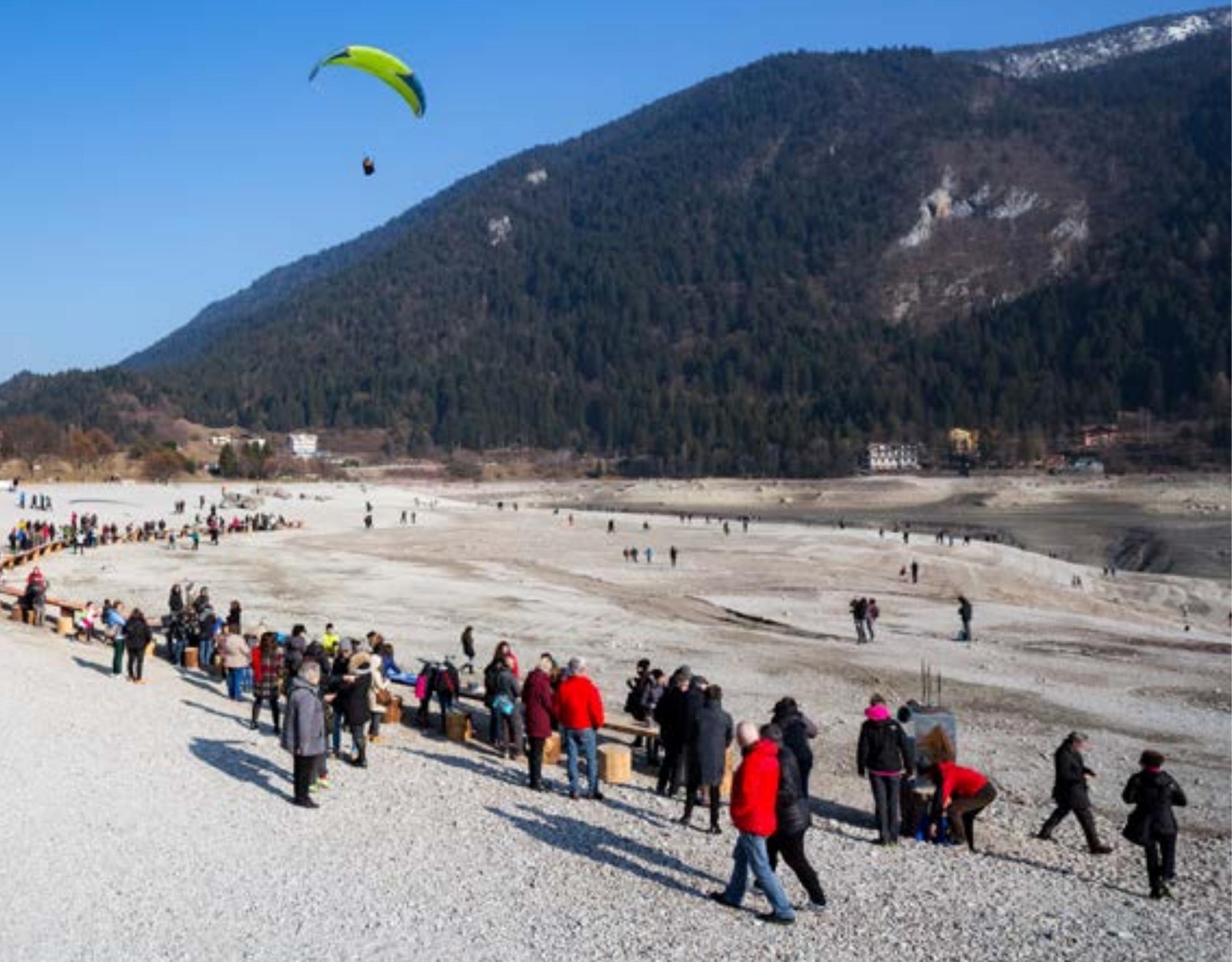


una ricostruzione di senso individuale e collettiva. Tale ricostruzione è possibile grazie all'intervento del Collettivo OP con il progetto "Io. Sempre. Opera dello svelamento della conoscenza e rinnovamento delle acque del Lago di Molveno".

Gli interventi dell'opera sono molteplici, oggi raccontati in un pregevole catalogo che testimonia il successo dell'iniziativa (Curti, Gasperi, Grigolli 2018), e tutti concorrono a "cambiare il punto di vista". Il tavolo dell'interazione (un asse in legno lungo 100 metri installato sulle sponde per favorire occasioni di incontro, condivisione e socialità), la scultura di MOG (tre elementi modulari in ferro e acciaio che rappresentano il lago in verticale), il manifesto poetico di Alessandro Cremonesi, i paesaggi sonori di Luca Lagash (un percorso onirico di suoni provenienti da impianti installati sul fondale). Tutti ci introducono ad una percezione diversa, che
Figg. 2-3

"Dentro Fuori" di Michele De Lucchi,

l'installazione è

stata inaugurata nel giugno del 2018

(foto Giacomo

Bianchi/@Arte Sella)

Fig. 4

La scultura monumentale di Morgana Orsetta Ghini (MOG) (foto Nicola Gnesi)

Fig. 5 La grande M della Michelin nel parco del quartiere Albere

a Trento vista dal promontorio di Sardagna (foto Anna Scalfi).
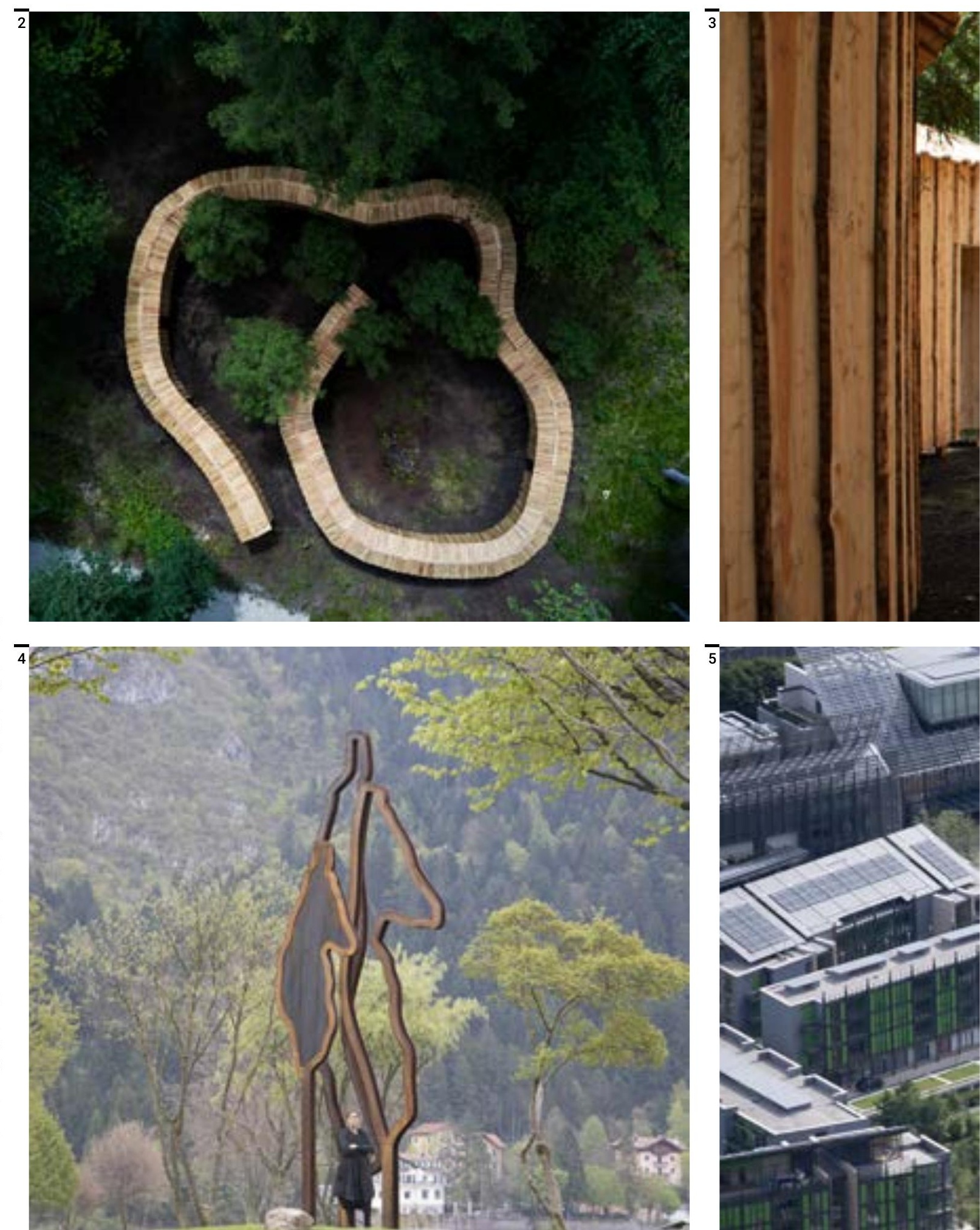
non si fonda esclusivamente sulla vista di un luogo, ma ci invitano soprattutto ad un attraversamento: il lago non è più percepito da un occhio separato e distante ma da un corpo che cammina. In questo senso la percezione è l'azione di un corpo in movimento che sente e che ci suggerisce modi altri di pensare e vivere il paesaggio (Mallgrave, 2015).

Il progetto ci consegna molte riflessioni relative alla percezione ma anche alla temporaneità e all'essenzialità di ogni paesaggio. Il paesaggio non è fatto per essere né venduto e né comprato, anche se può essere una risorsa con importanti ricadute economiche, ma è fatto innanzitutto per essere vissuto. Il transito "dal visto al vissuto" richiede il superamento di una visione esclusivamente decorativa, come realtà "eccezionale" da ammirare "là fuori", per arrivare ad un'idea che connette il paesaggio alla vivibilità. L’esperienza del pae-
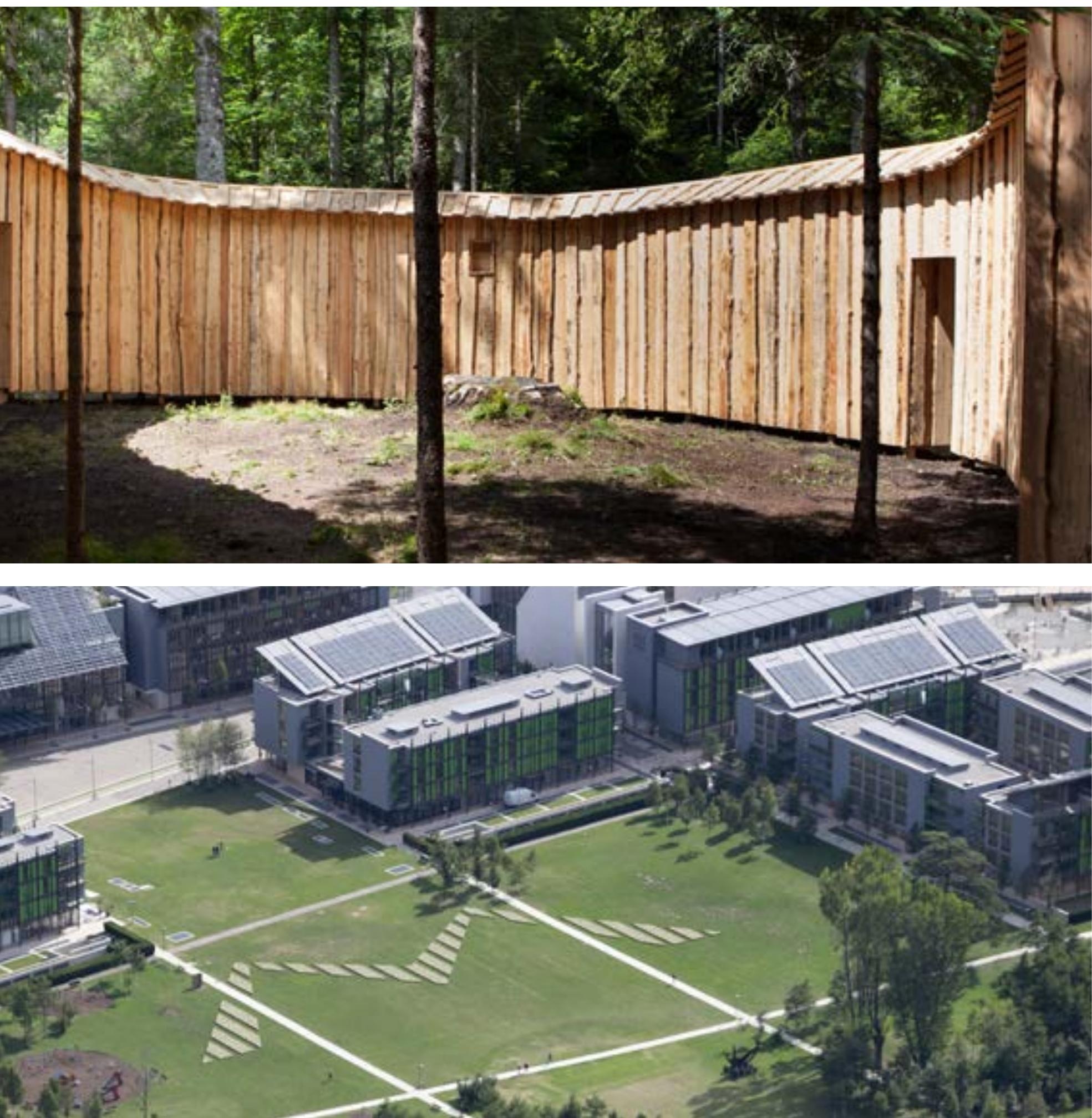
saggio, infatti, è stata spesso interpretata attraverso un sistema di percezione centrato sulla vista e caratterizzato dalla frontalità e dalla distanza di un individuo-spettatore (Vitale, 2015). La frontalità garantisce la rettitudine dello sguardo che si posa sull'oggetto esterno e separato. La distanza permette di sottrarsi alla confusione dell'immedesimazione ottenendo obiettività e possibilità di una fruizione contemplativa. L'idea che il lago sia qualcosa che ci sta davanti, preferibilmente da contemplare assorti seduti su una panchina, si fonda su una logica cartesiana che tende a separare corpo e mente, natura e cultura, osservatore e osservato, interno ed esterno, pieno e vuoto, visibile e invisibile. Il superamento del modello di un uomo che vive con "intorno" la natura e che "guarda" il paesaggio può avvenire solo attraverso la presa di distanza da qualsiasi forma di dualismo: non vi è mai un paesaggio da un lato e una percezione di un soggetto dall'altro. Dovremmo così imparare a pensarci come "parte del tutto" e come "naturalmente culturali", frutto di un'inestricabile intreccio tra natura e cultura (Morelli, 2011). Il lavoro del Collettivo OP ci aiuta a riconoscere la reciproca fecondazione tra natura e cultura. Per questo i paesaggi della nostra vita, come il lago di Molveno, sono sempre temporanei, provvisori, con una propria storia evolutiva.

\section{Sotto/Sopra. "Fiori dalla fabbrica" di Anna Scalfi Eghenter}

Sono circa 20.000 i tulipani bianchi fioriti nell'area dove una volta sorgeva l'azienda Michelin, multinazionale produttrice di pneumatici che ha segnato per oltre settant'anni la storia della città di Trento, dal 1927 fino alla sua chiusura nel 1999. A piantare i bulbi, nel mese di ottobre 2018, sono accorsi migliaia di cittadini, alunni delle scuole, famiglie con bambini, rappresentanti di associazioni della società civile, ex lavoratori della fabbrica. I bulbi disegnano sul terreno la forma del simbolo che era saldato sul cancello della fabbrica: la sagoma di una grande "M", appunto come Michelin, occupa l'area su cui una volta sorgeva l'industria. L'angolo da cui inizia la "M", in basso a sinistra, coincide infatti con quello da cui partiva lo stabilimento cancellato da un intervento di rigenerazione urbana che ha portato alla costruzione del nuovo quartiere delle Albere progettato da Renzo Piano.

"Fiori dalla fabbrica (Trento, Michelin 19271999)" di Anna Scalfi Eghenter è prima di tutto un progetto di arte partecipata, un'azione collettiva che vuole promuovere innovazione sociale e ricordarci che la stessa memoria è un processo socialmente costruito. Una memoria intima, come quella degli ex operai della fabbrica, ma allo stesso tempo collettiva, patrimonio di una comunità che ha fondato sul lavoro il proprio percorso di emancipazione. Con il riaffiorare dei tulipani emerge dalla memoria il senso della sacralità del lavoro: uomini e donne che per anni hanno vissuto insieme nello stesso luogo, recandosi in fabbrica alla stessa ora, svolgendo spesso lo stesso compito, condividendo la vita quotidiana. Una sacralità persa nell'epoca in cui le nuove tecnologie informatiche e la robotizzazione hanno decretato "la fine del lavoro" (Rifkin, 1995), o almeno la fine del lavoro organizzato così come l'avevamo conosciuto per i molti anni dell'epoca industriale.

Un evento architettonico non è mai limitato al tempo della sua realizzazione materiale, al tempo in cui si progetta e si costruisce, comincia prima e soprattutto "continua dopo, nell'uso nelle trasformazioni che subisce, nelle memorie che suscita e che si trasferiscono in altri eventi che seguiranno" (De Carlo, 1981). "Fiori dalla fabbrica" costituisce un'architettura virtuale e leggera quasi in contrapposizione con l'imponenza e la pesantezza di quella struttura industriale che rievoca; nello stesso tempo si pone però in stretta relazione con il nuovo contesto del parco cittadino, in particolare con la trama dei camminamenti, che diventa così parte integrante dell'opera stessa. Una straordinaria sintesi tra la rievocazione della dimensione storica e la conversazione con la dimensione contemporanea.

Superato l'assalto dei conigli ai bulbi durante i mesi invernali, i fiori bianchi dei tulipani a forma di calice splendono al sole, ma poi quando cominceranno ad appassire e le foglie a seccarsi, il progetto avrà svolto il suo compito raccontando di un passato di lavoro nobile e ricucendo una continuità ideale tra storia e presente.

L'istallazione è stata accompagnata da molte attività parti integranti dell'iniziativa: presentazioni pubbliche, interventi nelle scuole, incontri tra ex operai e giovani, un seminario universitario sulla pratica artistica, la produzione di un'audioguida con le voci di chi lavorava e conosceva la fabbrica. Attività che incrementano la dimensione educativa e partecipativa del progetto e lo rendono occasione per il recupero del valore culturale e sociale di un luogo che ha segnato indelebilmente la storia di una città.

\section{Dentro/Fuori. Michele De Lucchi ad Arte Sella}

Arte Sella da più di trent'anni rappresenta un'esperienza internazionale unica per la capacità di fondere l'arte con la natura e per favorire l'ibridazione di codici e linguaggi diversi accomunati dall'intessere verso il continuo dialogo tra la creatività e il mondo naturale. Artisti provenienti da tutto il mondo, del calibro di Giuliano Mauri, Nils-Udo, John Grade e Michelangelo Pistolet- 
to, hanno realizzato opere che definiscono, lungo un sentiero forestale sul monte Armentera, il percorso ArteNatura.

Nel 2016 è stata aperta, nel giardino di Villa Strobele, dimora storica e luogo di nascita di Arte Sella, una sezione dedicata all'architettura nella quale nel giugno del 2018 Michele De Lucchi ha realizzato l'opera "Dentro Fuori". L'opera richiama l'immagine di un muro, costruito interamente in larice proveniente dalla Valsugana, che dispone di una copertura in scandole realizzate con la tradizionale tecnica a spacco. Nelle parole dell'autore l'installazione non vuole creare ostacoli: “è un'architettura libera e libera la mente di chi la percorre. Si muove intorno agli alberi senza intaccarli con la sua presenza, ma includendoli al suo interno".

"Dentro Fuori" è un muro a forma di spirale che nasce all'aperto tra la natura ma che nello stesso tempo definisce lo spazio aperto, con le sue fenditure, sapientemente inserite nel produrre un gioco di luci e ombre, restituisce un significato ambiguo rispetto al tradizionale immaginario concettuale di un muro che definisce, demarca e separa. $\mathrm{Al}$ centro dell'opera vi è quindi l'archetipo del muro reinterpretato nella sua ambiguità quale elemento in grado di frazionare lo spazio attraverso la de- finizione di confini rigidi e definiti, ma nello stesso tempo capace di regalare riparo e protezione. Il muro "nella natura esalta la natura stessa, la rende più evidente, più amichevole, più a contatto con le proporzioni dell'uomo". I varchi e gli squarci presenti sulla superficie attivano connessioni tra interno ed esterno, trasformano il muro in cornice, una finestra sul paesaggio. L'architettura di De Lucchi ci rimanda alla necessità di una "epistemologia del confine" (Tagliagambe, 1997) che si impegna a pensare in maniera congiunta le forme e gli eventi tradizionalmente visti come separati, e così il muro sembra più un'interfaccia che crea una via di comunicazione piuttosto che una struttura che funge da barriera.

Solo dopo pochi mesi dalla sua inaugurazione, nell'ottobre del 2018, l'opera è stata seriamente danneggiata da un evento calamitoso: una tempesta di vento senza precedenti ha flagellato $\mathrm{i}$ boschi di molte montagne delle Alpi nord-orientali. Un'opera effimera, destinata sin dalla nascita a degradare secondo i tempi e i ritmi naturali, ci aiuta ancor di più a riflettere sul modo di pensare la progettazione architettonica anche in relazione al riscaldamento globale e agli effetti che l'accelerazione delle attività antropiche non più sostenibili hanno sui nostri spazi di vita.

\section{Bibliografia}

Curti Serena, Gasperi Maura, Grigolli Paolo (a cura di), (2018), lo. Sempre. Opera dello svelamento, conoscenza e rinnovamento delle acque del Lago di Molveno, Comune di Molveno, Trento.

De Carlo Giancarlo (1981), «Del ribaltamento del termine riuso nella prassi architettonica», in Di Biasi Carolina, Donati Lucia, Fontana Carlotta, Paolillo Pier Luigi (a cura di), Riuso e riqualificazione edilizia negli anni '80, Franco Angeli, Milano, p. 384.

De Rossi Antonio (2018), «Introduzione. L'inversione dello sguardo. Per una nuova rappresentazione territoriale del paese Italia», in De Rossi Antonio (a cura di), Riabitare I'Italia, Donzelli, Roma, pp. 3-18.

Mallgrave Harry Francis (2015), L'empatia degli spazi. Architettura e neuroscienze, Raffaello Cortina Editore, Milano. Morelli Ugo (2011), Mente e paesaggio. Una teoria della vivibilità, Bollati Boringhieri, Torino.

Rifkin Jeremy (1995), La fine del lavoro, Baldini\&Castoldi, Milano.

Tagliagambe Silvano (1997), Epistemologia del confine, II Saggiatore, Milano.

Vitale Sergio (2015), Il paesaggio e il suo rovescio. Distanza e proiezione nel luogo dell'Altro, Editrice Clinamen, Firenze. 